Because of the nonlinear features of the chemical reactions involved, a whole branch of mathematics has arisen to model the complex behaviour of solids, liquids and gases which are undergoing combustion. This, coupled with great advances in numerical approaches, has led to the formation of a new journal specifically devoted to the "application of mathematical theory, modelling, numerical simulation and experimental techniques to the study of combustion". The inclusion of experimental techniques in its scope is laudable and, it is hoped, will encourage greater involvement of experimentalists in the journal.

Combustion Theory and Modelling has been published since March 1997 and has received papers on a wide area of topics, such as flame stability, turbulent combustion, thermal explosion, compressible reacting flows, detonations and filtration combustion. In that a growing number of authors are making use of the journal, it is evident that it is taking a leading place in the status of archived combustion literature. Furthermore, it is readily available at competitive prices for personal subscription and, most importantly, for library subscription. It is the relative cheapness of library availability that should be most attractive in the present financial climate of universities in the UK and world-wide.

The high profile of theory and modelling in this quarterly is particularly welcome. The study of combustion fundamentals is vital to making progress in our understanding of practical combustion systems. Too often the tendency in research has been to regard theory as either too difficult, or naively to regard computation as somehow having replaced the need for modelling altogether. Advance in any scientific research area is connected to the progress of experiment (numerical or laboratory) coupled with testable theory. That way physical principles are uncovered and light the way for further advance.

A. C. McIntosh is in the Department of Fuel and Energy, University of Leeds, Leeds LS2 9JT, UK.

\section{Golden age journal}

Multibody System Dynamics

Editor Werner Schiehlen

Kluwer Academic Publishers. 4/yr. \$241.50

(institutional), $\$ 85$ (personal)

\section{John Hogan}

Contemporary mechanics traces its origins to Newton's laws of motion. Traditionally divided into fluid and solid mechanics, the theoretical side in the early part of this century saw ideas such as boundary layer theory give analytic tractability to the otherwise impenetrable continuum equations in these areas. Enormous strides in computer power mean that problems such as the large-scale computation of global weather patterns can now be addressed. More recently, the qualitative approach crucial to understanding nonlinear dynamics (including 'chaos theory') has been embraced by mechanics to the extent that the subject may be on the verge of a golden age, in which fields previously thought impenetrable can be explored.

The international body in the subject is the International Union of Theoretical and Applied Mechanics (IUTAM). Its president, Werner Schiehlen, is the editor of Multibody System Dynamics, which is devoted to one of the most important new fields. Examples of such systems include nearly every man-made object of engineering importance, such as cars, lorries, railway vehicles and robots.

Looking through the articles in this journal, it is clear that the equations of motion can be written down, that computers can produce solutions and that, given enough parameters, sufficient agreement can be found with observation. But something is missing. There seems to be no visceral, intuitive understanding of the behaviour of such systems - with their enormous numbers of degrees of freedom - especially when subject to constraints. Of course this is a highly non-trivial problem, but without a 'feel' for a problem, progress is liable to be restricted. The success and even survival of the journal may well be directly related to this issue.

Nevertheless, Schiehlen is to be congratulated for highlighting this area, and Kluwer applauded for its faith in him. Readers of Nature interested in this field should start with Schiehlen's comprehensive review in Multibody System Dynamics 1, 149-188 (1997), and then attend IUTAM's congress in Chicago in August 2000.

John Hogan is in the Department of Engineering Mathematics, Queen's Building, University Walk, Bristol BS8 1TR, UK.

\section{Living on the edge}

\section{Extremophiles: Life Under Extreme Conditions}

Editors Koki Horikoshi, Garo Antranikian, William D. Grant, Terry A. Krulwich and Juergen Wiegel

Springer. 4/yr. \$267, ¥25,000, DM380

\section{John A. Baross}

Why do we suddenly need a journal dedicated to micro-organisms that grow under conditions we humans define as extreme? Micro-organisms that grow in saturated concentrations of salt have been studied for almost 80 years. Pressure- and cold-loving bacteria were identified from the deepest ocean trenches during the Danish Galathea expedition in 1952. For at least 20 years we have known of micro-organisms that grow at temperatures close to $100{ }^{\circ} \mathrm{C}$, alkalinities exceeding $\mathrm{pH} 10$, and acidities near $\mathrm{pH} 0$.

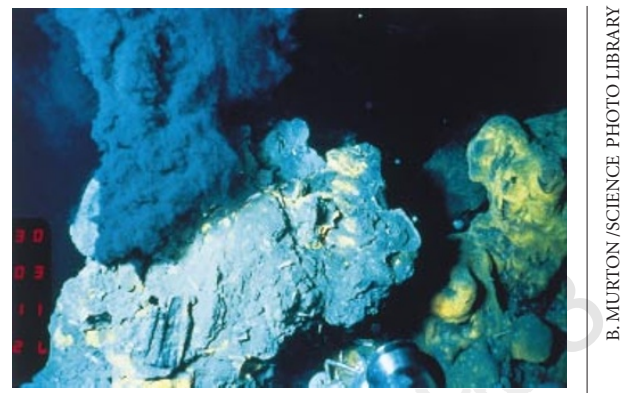

The Saracen's Head 'black smoker' vent in the Atlantic Ocean floor.

Yet over the past ten years extremophiles have taken on a life of their own, no longer studied as academic peculiarities. High- and low-temperature enzymes from thermal extremophiles are exploited for their many biotechnological applications, while acidophiles assist in the mining of precious metals from complex minerals. Global phylogenetic trees based on nucleic acid sequences indicate that hyperthermophiles are the oldest of extant organisms, holding potential clues to the origin of life on Earth. Their physiologies suggest that they could grow on other solar bodies or survive transit between them. Such controversial ideas, spurred most recently by evidence that the Jovian moon, Europa, may have a liquid-water ocean, are very much part of the study of extremophiles and the emergent field of 'astrobiology'. Life in extreme environments is in vogue.

Against this backdrop, the creation of a journal dedicated to extremophiles was inevitable. Its appearance is also an acknowledgement of the growing community of researchers dedicated to studying these very exciting organisms from a wide range of disciplinary perspectives. This growth is reflected in the diversity of research papers in the first four issues reporting novel and important new discoveries. The topics range from descriptions of new organisms and physiologies to the characterization of their enzymes and molecular biology. One can also learn that the highest temperature for growth by a pure culture has risen from 110 to $113^{\circ} \mathrm{C}$, that there exist organisms that can oxidize hydrogen gas using ferric-iron as the electron acceptor, and that physiologically diverse heterotrophs exist in the deepest trenches of the ocean.

Overall, the research articles in the first four issues are of high quality. Three issues also offered a very useful review article: I recommend that the editors continue to solicit reviews that keep the community informed of current trends in this rapidly changing field. This attractively produced journal is essential reading for anyone interested in life in extreme environments and its ramifications to other fields of inquiry.

John Baross is in the School of Oceanography,

University of Washington, Box 357940, Seattle, Washington 98195, USA. 